\title{
Potential Toxicity of Medicinal Plants Inventoried in Northeastern Morocco: An Ethnobotanical Approach
}

\author{
Loubna Kharchoufa $^{1}$, Mohamed Bouhrim ${ }^{1}$, Noureddine Bencheikh ${ }^{1}{ }^{(D}$, Mohamed Addi $^{2}\left(\mathbb{D}\right.$, Christophe Hano $^{3}{ }^{(}$, \\ Hamza Mechchate ${ }^{4, *}$ (D) and Mostafa Elachouri ${ }^{1}$ \\ 1 Laboratory of Bioresources, Biotechnology, Ethnopharmacology and Health, URAC-40, Department of \\ Biology, Faculty of Sciences, Mohammed First University, Oujda 60040, Morocco; \\ khloubna43@gmail.com (L.K.); mohamed.bouhrim@gmail.com (M.B.); \\ bencheikh_noureddine1718@ump.ac.ma (N.B.); elachourimostafa@gmail.com (M.E.) \\ 2 Laboratoire d'Amélioration des Productions Agricoles, Biotechnologie et Environnement, (LAPABE), \\ Faculté des Sciences, Université Mohammed Premier, Oujda 60000, Morocco; m.addi@ump.ac.ma \\ 3 Laboratoire de Biologie des Ligneux et des Grandes Cultures, INRAE USC1328, Campus Eure et Loir, \\ Orleans University, 45067 Orleans, France; christophe.hano@univ-orleans.fr \\ 4 Laboratory of Biotechnology, Environment, Agrifood and Health, Faculté des Sciences Dhar el Mahraz, \\ University of Sidi Mohamed Ben Abdellah, Fez 30050, Morocco \\ * Correspondence: Hamza.mechchate@usmba.ac.ma
}

\section{check for} updates

Citation: Kharchoufa, L.; Bouhrim, M.; Bencheikh, N.; Addi, M.; Hano, C.; Mechchate, H.; Elachouri, M. Potential Toxicity of Medicinal Plants Inventoried in Northeastern Morocco: An Ethnobotanical Approach. Plants 2021, 10, 1108. https://doi.org/ 10.3390/plants10061108

Academic Editors: Luigi Milella and Mariangela Marrelli

Received: 15 April 2021

Accepted: 28 May 2021

Published: 31 May 2021

Publisher's Note: MDPI stays neutral with regard to jurisdictional claims in published maps and institutional affiliations.

Copyright: (c) 2021 by the authors Licensee MDPI, Basel, Switzerland. This article is an open access article distributed under the terms and conditions of the Creative Commons Attribution (CC BY) license (https:// creativecommons.org/licenses/by/ $4.0 /)$.

\begin{abstract}
Herbal medicine and its therapeutic applications are widely practiced in northeastern Morocco, and people are knowledgeable about it. Nonetheless, there is a significant knowledge gap regarding their safety. In this study, we reveal the toxic and potential toxic species used as medicines by people in northeastern Morocco in order to compile and document indigenous knowledge of those herbs. Structured and semi-structured interviews were used to collect data, and simple random sampling was used as a sampling technique. Based on this information, species were collected, identified, and herbarium sheets were created. The collected data were analyzed using two quantitative indices: informant consensus factor (ICF) and fidelity level (Fl), as the degree of these indices give an insight into the level of toxicity of a given plant. The results revealed the knowledge of 55 species belonging to 36 families. The most represented families were Apiaceae, Asteraceae, Solanaceae, and Fabaceae. Furthermore, the majority of the species cited were herbs $(67 \%)$, and the most common toxic parts were seeds, followed by leaves and roots. According to the informant consensus factor, death $(0.81 \%)$ had the highest agreement, followed by the urological $(0.76 \%)$ and skin $(0.75 \%)$ categories. The most significant plants in terms of fidelity level were Solanum sodomaeum L. and Nerium oleander L. for death, Arisarum vulgare O. Targ. Tozz., Mentha spicata L., and Morus alba L. for the digestive category, Petroselinum crispum (Mill.) Fuss. and Citrus $x$ aurantium L. for cardiovascular category, Urtica dioica L. for skin category, Datura stramonium L., and Ephedra altissima Desf. for neurological category, and finally Crocus sativus L. for general and unspecified category. This work highlights a valuable traditional knowledge of poisonous and potential poisonous plants in northeastern Morocco. Further phytochemical and toxicological research is needed to determine the safety of these prized herbs.
\end{abstract}

Keywords: ethnobotany; traditional medicine; toxic plants; toxicity; Africa

\section{Introduction}

Over the last decade, there has been a great revival of reliance on herbal product medication to manage various ailments [1-3]. According to the World Health Organization (WHO), 80\% of the world's population, especially people in developing countries, is dependent on traditional medical practices for some aspect of primary health care [4]. In Morocco, the use of plants for medical care has been practiced since time immemorial and patients rely more on folk medicine: it is estimated that between $50 \%$ and $75 \%$ of the local population relies on traditional Moroccan remedies [5-9]. 
Like other regions in Morocco, the people of the northeastern region of the country are more concerned with the use of herbal medicine, and many of them have been collecting and preparing plants for medicinal purposes and the relief of disease symptoms [10,11]. However, the widespread belief that herbal drugs are perfectly safe and devoid of adverse effects is untrue and misleading $[12,13]$. Herbs can cause a wide range of undesirable or adverse reactions, some of which can result in severe injuries, life-threatening conditions, and even death [14]. Despite the popularity of these herbal products and their widespread use, the scientific evidence and assessment of the safety and toxicity of medicinal plants has been largely ignored, with the exception of a recent bibliographic work published by our team in which we documented a list of 89 toxic plants used traditionally by people living in this area [15]. In this regard, we conducted this fieldwork in order to assess local people's knowledge of the toxicity of medicinal plants. To the best of our knowledge, the current study is the first to deal with the ethnotoxic knowledge of herbs. Throughout this work, we attempt to document and analyze traditional knowledge about toxic plants used as medicine by people in northeastern Morocco using both qualitative and quantitative approaches including various indexes such as informant consensus factor (ICF) and fidelity level (Fl).

\section{Results and Discussion}

\subsection{Demographic Profile of the Informants}

The demographic features of the informants were determined and recorded in this ethnobotanical survey (Table 1). The results revealed that 386 informants (345 were local inhabitants and 41 were herbalists) in the study zone belonged to three various northeastern Moroccan districts including $41.70 \%$ of Oujda Prefecture, $32.90 \%$ of Berkane Province, and $25.38 \%$ of Jerada Province. Of all interviewed persons, $67.61 \%$ were females, and $32.38 \%$ were males. The women's responsibilities to care for all family members (husband and children) including nutrition and medication, and the fact that they were most often at home, could explain the high percentage observed $(67.61 \%)$. These observations were consistent with several studies conducted in Morocco [6,7,10]. In terms of age, we found that out of the 386 participants, 56 persons (14.50\%) were less than 30 years, $241(62.43 \%)$ were between the ages of 30-60 years, $74(19.17 \%)$ were between the ages of $60-80$ years, and $15(3.88 \%)$ were over the age of 80 years. Similar results have been reported in several studies $[16,17]$. Previous research has shown that the practice of phytotherapy is always more important for the elderly than for the young $[18,19]$. As a result, people of this age are more often responsible for the health of their families [20]. During the course of the study, it was observed that $43.00 \%$ of the participants were illiterate, $25.64 \%$ had completed primary school, $16.83 \%$ had completed university, and $14.50 \%$ had completed high school. These findings corroborate those obtained in various Moroccan ethnobotanical studies [21,22]. People with a lower educational level, in fact, have more expertise in the field of traditional medicine and medical folk botany [23]. Regarding the participants' habitat, $60.10 \%$ were living in rural communities, and $39.10 \%$ were living in urban areas. These results are consistent with those previously reported in oriental Morocco [10]. Bellakhdar [24] indicates that rural participants are the primary consumers of medicinal plants because the rural suppliers maintained close contact with nature, which still provides them with many resources. 
Table 1. Informant socio-demographic characteristics.

\begin{tabular}{ccc}
\hline Socio-Demographic & \multicolumn{2}{c}{ Sample } \\
Variables & Number & Percentage (\%) \\
\cline { 2 - 3 } & & \\
District & 161 & 41.70 \\
Oujda & 137 & 32.90 \\
Berkane & 88 & 25.38 \\
Jerada & & \\
Function & 345 & 89.37 \\
Ordinary inhabitant & 41 & 10.63 \\
Herbalists & & \\
Habitat & 154 & 39.89 \\
Urban & 232 & 60.10 \\
Rural & & \\
Age (Years) & 56 & 14.50 \\
$<30$ & 241 & 62.43 \\
30-60 & 74 & 19.17 \\
60-80 & 15 & 3.88 \\
>80 & & \\
Gender & 125 & 32.38 \\
Male & 261 & 67.61 \\
Female & & \\
Study Level & 166 & 43 \\
Illiterate & 99 & 25.64 \\
Primary & 56 & 16.83 \\
High school & 65 &
\end{tabular}

\subsection{Floristic Characteristics of Poisonous Plants}

The present ethnobotanical survey revealed 55 toxic and potential toxic herbs from 36 botanical families and 36 genera. The plant family with the highest number of species was Apiaceae, which contributed six species (11\%), followed by the Solanaceae and Asteraceae families, which contributed four species each $(7 \%)$, and the Fabaceae family, which contributed three species (5\%) (Figure 1). Otherwise, the Amaranthaceae, Brassicaceae, $\mathrm{Cu}$ curbitaceae, Euphorbiaceae, Lamiaceae, and Rutaceae families each have two species (4\%), and the remaining 26 families each have only one species (2\%). Plant taxonomy at the family level is an important factor in determining the usefulness of plant species to local people. Some plant families are more useful than others in certain application categories [25]. The same logic applies to toxic and potential toxic species [26]. The Apiaceae, Asteraceae, Solanaceae, and Fabaceae families have been identified as the leading families of plants in our study area. This is consistent with our previous study in northeastern Morocco, where we identified 89 plants distributed across 45 families, the most dominant of which were Apiaceae, Fabaceae, Asteraceae, Brassicaceae, Lamiaceae, and Solanaceae [15]. A study conducted in India concluded that species belonging to the Fabaceae, Asteraceae, Apocynaceae, Solanaceae, and Euphorbiaceae families were frequently cited as toxic by local residents [17]. The toxicity of these families has been attributed to the presence of specific compounds such as acrid substances, alkaloids, heterosides, photosensitizing compounds, saponins, or volatile oils in Asteraceae; tropane, pyrrolidine, and pyrrolic alkaloids, protoand glycol-alkaloids, or cardenolides in Solanaceae; and alkaloids, coumarin, heterosides, saponins, photosensitizing compounds, or selenium in Fabaceae [17,27]. Among the plants collected, herbs were the most common and represented $67 \%$ taxa, followed by shrubs (18\% taxa) and trees (15\% taxa). These results are consistent with previous studies [17]. Among the different toxic parts, seed toxicity was the highest $(30 \%)$, followed by leaf toxicity $(19 \%)$, root toxicity $(14 \%)$, bulb toxicity $(8 \%)$, aerial part toxicity $(7 \%)$, fruit toxicity $(6 \%)$, stem toxicity $(6 \%)$, leaf stem toxicity $(3 \%)$, and whole plant toxicity (3\%) (Figure 2 ). 
These reports are in contrast with the previous study revealing the predominance of whole plant toxicity [28].

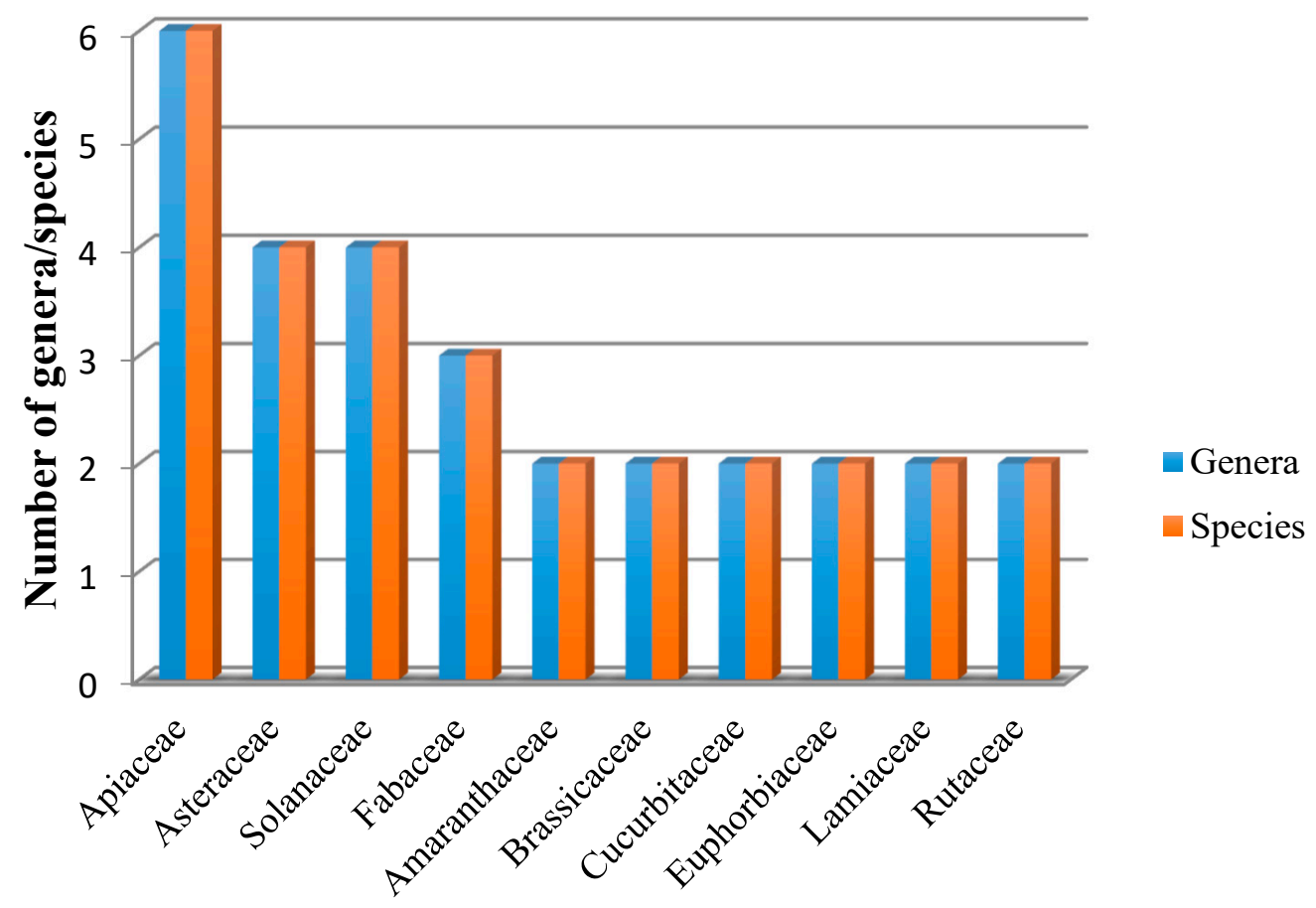

Family

Figure 1. The main families of plants.
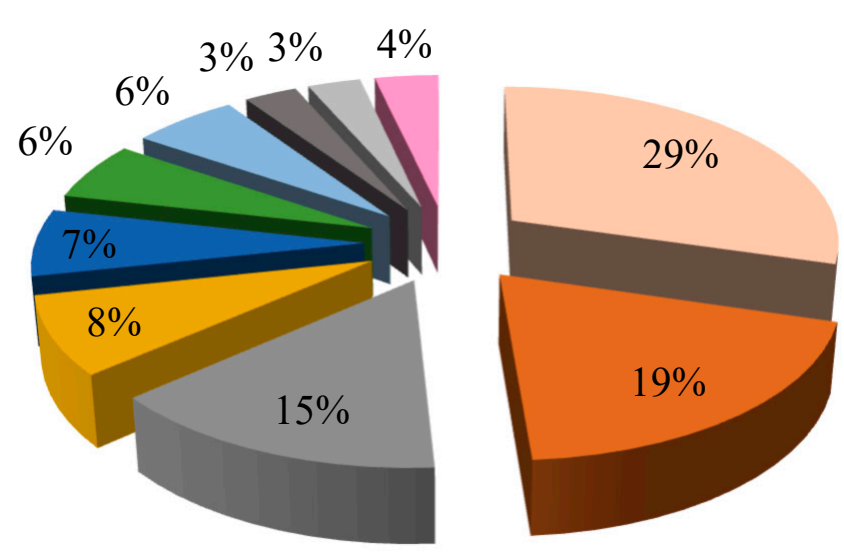

Seed

- Leaf

Root

Bulb

- Aerial part

- Fruit

Stem

Leaf stem

$\square$ Whole plant

$\square$ Others

Figure 2. Percentage of the different parts.

\subsection{Traditional Knowledge of Poisonous Herbs}

The documented poisonous and potentially poisonous plants and their ethnomedicinal data, along with a local name, life form, traditional uses, route of administration, poisonous part, and toxic signs, are summarized in Table 2. Our ethnobotanical investigation reached 386 participants in northeastern Morocco, and 148 persons responded. Percentage knowledge of poisonous and potential poisonous plants was $38.34 \%$ within the study population. A total of 55 medicinal plant species have been reported to be toxic or to have some toxicity. Twenty-six of these species were mentioned by fewer than three informants each. The remaining 29 species were mentioned by at least three different informants. 
Ethnobotanical information showed that all of the selected species have toxic effects such as neurological (dizziness, delirium, madness, hallucination, migraine, headache, sedation, loss of consciousness, sleep disturbance), digestive (vomiting, diarrhea, gastroenteritis, constipation, nausea, indigestion, stomachache, bloating, abdominal pain, mouth, bile problems, jaundice, gallbladder), skin (skin irritation, itchy, inflammation, skin problems), Urological (kidneys problems, diuretic, bladder diseases), cardiovascular (cardiac problems, hypertension, hypotension), and female genitals (abortion, sterility). Among the 55 species collected from the various northeastern Moroccan regions, Nerium oleander L., Carlina gummifera (L.) Less., Cannabis sativa L., Peganum harmala L., Allium sativum L., Aristolochia fontanesii Boiss. \& Reut., Trigonella foenum-graecum L., Glycine max (L.) Merr., Daphne gnidium L., and Ricinus communis L. were the most cited plant species. According to the results presented in our previous study, Nerium oleander L., Carlina gummifera (L.) Less., Aristolochia fontanesii Boiss. \& Reut., and Ricinus communis L. are all considered as severe poisonous plant species [15,29-31].

\subsection{Quantitative Analysis of Data}

To quantify our data, we used two ethnobotanical indexes adapted to toxicological studies: the informant consensus factor (ICF), which indicates the agreement about the toxicity of plant species, and the fidelity level (Fl), which indicates the most important species causing a particular illness.

\subsubsection{Informant Consensus Factor (ICF)}

Based on the major toxicity effects, we classified the plant species listed in the present study into 12 groups using the International Classification of Primary Care- $2^{\text {nd }}$ Edition (ICPC-2) [32]. Our finding indicated that the value of the informant consensus factor (ICF) ranged from 0 to 0.81 (Table 3 ). The elevated value was assigned to death (0.81), with 94 toxic citations for 18 herbs, followed by urological (ICF $=0.76$; 14 toxic reports, four species), neurological (ICF $=0.73 ; 76$ toxic reports, 21 species), digestive (ICF = 0.67; 95 toxic reports, 32 species), skin (ICF $=0.66 ; 10$ toxic reports, four species), cardiovascular $(\mathrm{ICF}=0.63 ; 31$ toxic reports, 12 species $)$, eye $(\mathrm{ICF}=0.57$; eight toxic reports, four species $)$, female genitals (ICF $=0.47 ; 18$ toxic reports, 10 species) and fever, allergy, pain general category with a ICF of 0.30 (11 toxic reports, eight species). The elevated value of ICF for some categories reflected a high level of homogeneity in consensus regarding the toxicity of plants. The high ICF for death may indicate a significant interrelationship between informants for a poisonous plant that causes this category. A low agreement observed between the informants was noted for plants affecting endocrine, metabolic, and nutritional category, respiratory category and blood, blood forming organs, and immune mechanism categories with ICF value zero for each one. According to Saleh Al-Qur'an [28], the low ICF value observed in our study could be attributed to a lack of communication among people in various areas. 
Table 2. List of toxic plants cited toxic by people in northeastern Morocco.

\begin{tabular}{|c|c|c|c|c|c|c|c|}
\hline $\begin{array}{c}\text { Family } \\
\text { Scientific Name }\end{array}$ & Local Name & $\begin{array}{c}\text { Herbaceous Life } \\
\text { Form/Voucher Number }\end{array}$ & $\begin{array}{c}\text { Rout of } \\
\text { Administration }\end{array}$ & Traditional Uses & Poisonous Part & Toxic Signs & NC \\
\hline \multicolumn{8}{|l|}{ Amaranthaceae } \\
\hline $\begin{array}{l}\text { Dysphania } \\
\text { ambrosioides (L.) } \\
\text { Mosyakin \& } \\
\text { Clemants }\end{array}$ & Mkhinza & Herb/HUMPOM571 & $\begin{array}{c}\text { Internal and external } \\
\text { uses }\end{array}$ & Headache, migraine, fever & $\begin{array}{c}\text { Leaf } \\
\text { Aerial part }\end{array}$ & $\begin{array}{l}\text { Madness, dizziness, death } \\
\text { (oral), gallbladder toxicity }\end{array}$ & 7 \\
\hline $\begin{array}{l}\text { Haloxylon scoparium } \\
\text { Pomel }\end{array}$ & Ramt & Herb/HUMPOM572 & Internal uses & Diabetes, scorpion bite & Aerial part & $\begin{array}{l}\text { Vomiting, abdominal pain, } \\
\text { pain general }\end{array}$ & 3 \\
\hline \multicolumn{8}{|l|}{ Amarillydaceae } \\
\hline Allium sativum $\mathrm{L}$. & Thouma & Herb/HUMPOM573 & & $\begin{array}{l}\text { Food, cold, hypertension, } \\
\text { asthma, cough, dental pain, } \\
\text { ear pain, digestive, antibiotic, } \\
\text { weight loss, hair } \\
\text { strengthening, immunity, } \\
\text { eczema, influenza, } \\
\text { hemorrhoids, gum disease }\end{array}$ & Bulb & $\begin{array}{l}\text { Hypotension, stomach-ache, } \\
\text { allergy, nephrotoxicity, } \\
\text { stomach burning }\end{array}$ & 22 \\
\hline Pistacia lentiscus L. & Dro/meska & Tree/HUMPOM574 & Internal uses & $\begin{array}{l}\text { Uterus cancer, diabetes, } \\
\text { gastro-intestinal disorders }\end{array}$ & Leaf, fruit & Constipation, skin irritation & 2 \\
\hline \multicolumn{8}{|l|}{ Apiaceae } \\
\hline Conium maculatum $\mathrm{L}$. & Ziyyata & Herb/HUMPOM575 & Internal uses & Cold & Fruit, seed & $\begin{array}{l}\text { Neurological problems, } \\
\text { abdominal pain, vomiting }\end{array}$ & 4 \\
\hline $\begin{array}{l}\text { Coriandrum sativum } \\
\text { L. }\end{array}$ & $\begin{array}{l}\text { Kosbare, } \\
\text { Kessbour }\end{array}$ & Herb/HUM-POM576 & $\begin{array}{c}\text { Internal and external } \\
\text { uses }\end{array}$ & $\begin{array}{c}\text { Food, mouth ulcers, frequent } \\
\text { urination }\end{array}$ & $\begin{array}{l}\text { Leaf, seeds, leafy } \\
\text { stem }\end{array}$ & $\begin{array}{l}\text { Forgetting, sleep, dizziness, } \\
\text { neurological problems, } \\
\text { allergy }\end{array}$ & 5 \\
\hline Ferula communis L. & $\begin{array}{l}\text { Kelkha } \\
\text { L-klekh } \\
\text { Fasoukh }\end{array}$ & Herb/HUMPOM577 & $\begin{array}{c}\text { Internal and external } \\
\text { uses }\end{array}$ & $\begin{array}{l}\text { Cold, food, magic, } \\
\text { anthelmintic, female sterility. }\end{array}$ & Stem, resin & $\begin{array}{l}\text { Abortion, irritation, death, } \\
\text { pain general, neurological } \\
\text { toxicity }\end{array}$ & 7 \\
\hline $\begin{array}{l}\text { Foeniculum vulgare } \\
\text { Mill. }\end{array}$ & Bassbass & Herb/HUMPOM578 & $\begin{array}{c}\text { Internal and external } \\
\text { uses }\end{array}$ & $\begin{array}{l}\text { Abdominal pain, intestine, } \\
\text { maternal milk. }\end{array}$ & Seed & $\begin{array}{l}\text { Dizziness, hypotension, } \\
\text { facial pills }\end{array}$ & 3 \\
\hline
\end{tabular}


Table 2. Cont.

\begin{tabular}{|c|c|c|c|c|c|c|c|}
\hline $\begin{array}{c}\text { Family } \\
\text { Scientific Name }\end{array}$ & Local Name & $\begin{array}{c}\text { Herbaceous Life } \\
\text { Form/Voucher Number }\end{array}$ & $\begin{array}{c}\text { Rout of } \\
\text { Administration }\end{array}$ & Traditional Uses & Poisonous Part & Toxic Signs & NC \\
\hline $\begin{array}{l}\text { Petroselinum crispum } \\
\text { (Mill.) Fuss }\end{array}$ & Maâdnousse & Herb/HUMPOM579 & $\begin{array}{l}\text { Internal and external } \\
\text { uses }\end{array}$ & Food, hypertension, hair & Leaf & Hypotension & 2 \\
\hline Thapsia garganica L. & Deryas & Herb/HUMPOM580 & Internal uses & $\begin{array}{l}\text { Antispasmodic, rheumatism, } \\
\text { women sterility, cough }\end{array}$ & Root & $\begin{array}{l}\text { Abortion (animal), vomiting } \\
\text { and diarrhea }\end{array}$ & 4 \\
\hline \multicolumn{8}{|l|}{ Apocynaceae } \\
\hline Nerium oleander L. & Dafla & Shrub/HUMPOM581 & External uses & $\begin{array}{l}\text { Stop nose bleeds, tonsillitis, } \\
\text { swollen gums, cold, headache, } \\
\text { teeth, rheumatism, sedative, } \\
\text { mumps, influenza, jaundice, } \\
\text { ear infections, mouth-ulcers, } \\
\text { diabetes, torticollis, irritable } \\
\text { bowel syndrome, skin } \\
\text { problems }\end{array}$ & Whole plant & $\begin{array}{l}\text { Death (oral), increased heart } \\
\text { rate and irregularity, toxic to } \\
\text { eyes, abdominal, pain, } \\
\text { stomachache, digestive } \\
\text { system disorders }\end{array}$ & 35 \\
\hline \multicolumn{8}{|l|}{ Araceae } \\
\hline $\begin{array}{l}\text { Arisarum vulgare } \mathrm{O} . \\
\text { Targ. Tozz. }\end{array}$ & Tabgouga & Herb/HUMPOM582 & $\begin{array}{c}\text { External and internal } \\
\text { uses }\end{array}$ & $\begin{array}{c}\text { Emetic, purgative, } \\
\text { dermatological problems }\end{array}$ & Tuber & $\begin{array}{c}\text { Mouth inflammation, } \\
\text { intestinal pain, vomiting, } \\
\text { hepatotoxicity }\end{array}$ & 2 \\
\hline \multicolumn{8}{|l|}{ Araliaceae } \\
\hline Hedera helix L. & Louwaya & Shrub/HUMPOM583 & External uses & Rheumatism, skin abscesses & Leaf, fruit & Death (oral) & 2 \\
\hline \multicolumn{8}{|l|}{ Aristolochiaceae } \\
\hline $\begin{array}{c}\text { Aristolochia } \\
\text { fontanesii Boiss. \& } \\
\text { Reut. }\end{array}$ & Baraztem & Shrub/HUMPOM584 & $\begin{array}{c}\text { External and internal } \\
\text { uses }\end{array}$ & $\begin{array}{l}\text { Cancer, skin infection, } \\
\text { intestinal parasites, diabetes }\end{array}$ & $\begin{array}{c}\text { Root, } \\
\text { whole plant }\end{array}$ & $\begin{array}{c}\text { Nephrotoxicity, } \\
\text { hepatotoxicity, vomiting, } \\
\text { dizziness, indigestion, death }\end{array}$ & 15 \\
\hline \multicolumn{8}{|l|}{ Asparagaceae } \\
\hline $\begin{array}{l}\text { Drimia maritima } \\
\text { (L.) Stearn }\end{array}$ & $\begin{array}{l}\text { Bes'al ed-dib } \\
\text { Bassila }\end{array}$ & Herb/HUMPOM585 & $\begin{array}{c}\text { External and internal } \\
\text { uses }\end{array}$ & $\begin{array}{l}\text { Detoxification, dental pain, } \\
\text { abdominal pain }\end{array}$ & Bulb & $\begin{array}{l}\text { Diarrhea, death, dizziness, } \\
\text { nausea, vomiting }\end{array}$ & 6 \\
\hline
\end{tabular}


Table 2. Cont.

\begin{tabular}{|c|c|c|c|c|c|c|c|}
\hline $\begin{array}{c}\text { Family } \\
\text { Scientific Name }\end{array}$ & Local Name & $\begin{array}{c}\text { Herbaceous Life } \\
\text { Form/Voucher Number }\end{array}$ & $\begin{array}{c}\text { Rout of } \\
\text { Administration }\end{array}$ & Traditional Uses & Poisonous Part & Toxic Signs & NC \\
\hline \multicolumn{8}{|l|}{ Asteraceae } \\
\hline $\begin{array}{c}\text { Carlina } \\
\text { gummifera (L.) Less. }\end{array}$ & Addad & Herb/HUMPOM586 & $\begin{array}{c}\text { External and internal } \\
\text { uses }\end{array}$ & $\begin{array}{l}\text { Headache, respiratory system, } \\
\text { sterility, rheumatism, skin } \\
\text { diseases, mouth pain, freckles } \\
\text { on the face, whiten the teeth, } \\
\text { facilitates childbirth, hair loss. }\end{array}$ & Root & $\begin{array}{l}\text { Vomiting, diarrhea, swilling, } \\
\text { death, digestive disorders }\end{array}$ & 25 \\
\hline $\begin{array}{l}\text { Artemisia arborescens } \\
\text { (Vaill.) L. }\end{array}$ & Chiba & Herb/HUMPOM588 & $\begin{array}{c}\text { External and internal } \\
\text { uses }\end{array}$ & Hypoglycemic, otitis & Aerial part & $\begin{array}{l}\text { Diarrhea, dizziness, } \\
\text { convulsion }\end{array}$ & 2 \\
\hline $\begin{array}{l}\text { Launaea arborescens } \\
\text { (Batt.) Murb. }\end{array}$ & Mmû-lbeyna & $\begin{array}{c}\text { Shrub } \\
\text { HUMPOM589 }\end{array}$ & Internal uses & $\begin{array}{l}\text { Hypoglycemic, respiratory } \\
\text { problems }\end{array}$ & Latex & Death & 2 \\
\hline \multicolumn{8}{|l|}{ Boraginaceae } \\
\hline Borago officinalis L. & $\begin{array}{l}\text { Lsan tawr } \\
\text { El hricha }\end{array}$ & Herb/HUMPOM590 & External uses & Knee pains & Root & Death (oral) & 2 \\
\hline \multicolumn{8}{|l|}{ Brassicaceae } \\
\hline $\begin{array}{c}\text { Anastatica } \\
\text { hierochuntica L. }\end{array}$ & $\begin{array}{l}\text { Lkmicha, } \\
\text { Keff-maryam, } \\
\text { Tamkelt }\end{array}$ & Herb/HUMPOM591 & Internal uses & Strengthen the uterus & Leafy stem & $\begin{array}{l}\text { Neurological disorders, } \\
\text { sleep disturbance }\end{array}$ & 2 \\
\hline \multicolumn{8}{|l|}{ Brassicaceae } \\
\hline Lepidium sativum $\mathrm{L}$. & Hab rachad & Herb/HUMPOM592 & $\begin{array}{l}\text { Internal and external } \\
\text { uses }\end{array}$ & $\begin{array}{c}\text { Hair, anemia, cough, intestinal } \\
\text { gas, strengthen hair, regulates } \\
\text { the menstrual cycle, maternal } \\
\text { milk }\end{array}$ & Seed & $\begin{array}{c}\text { Indigestion, diuresis, } \\
\text { abortion, dizziness } \\
\text { hepatotoxicity, hypertension }\end{array}$ & 8 \\
\hline
\end{tabular}


Table 2. Cont.

\begin{tabular}{|c|c|c|c|c|c|c|c|}
\hline $\begin{array}{c}\text { Family } \\
\text { Scientific Name }\end{array}$ & Local Name & $\begin{array}{l}\text { Herbaceous Life } \\
\text { Form/Voucher Number }\end{array}$ & $\begin{array}{c}\text { Rout of } \\
\text { Administration }\end{array}$ & Traditional Uses & Poisonous Part & Toxic Signs & NC \\
\hline \multicolumn{8}{|l|}{ Cannabaceae } \\
\hline Cannabis sativa $\mathrm{L}$. & Lkif & Herb/HUMPOM593 & External uses & $\begin{array}{l}\text { Hair loss, hair lengthening and } \\
\text { thickening, against dandruff, } \\
\text { strengthen hair, calming }\end{array}$ & Leaf, seed & $\begin{array}{l}\text { Ecstasy, hepatotoxicity } \\
\text { madness, sedation, } \\
\text { neurological toxicity, } \\
\text { respiratory problems, } \\
\text { dizziness, lose } \\
\text { consciousness, neurological } \\
\text { toxicity, death }\end{array}$ & 26 \\
\hline \multicolumn{8}{|l|}{ Caryophyllaceae } \\
\hline Herniaria cinerea DC. & Harasst lahjar & Herb/HUMPOM594 & Internal uses & Kidney stones & Aerial part & Hypertension & 2 \\
\hline \multicolumn{8}{|l|}{ Cucurbitaceae } \\
\hline $\begin{array}{l}\text { Bryonia cretica } \\
\text { subsp. dioica } \\
\text { (Jacq.) Tutin }\end{array}$ & Aneb dib & Herb/HUMPOM595 & $\begin{array}{c}\text { Internal and external } \\
\text { uses }\end{array}$ & $\begin{array}{l}\text { Hair loss, urinary tract } \\
\text { infection }\end{array}$ & Whole plant & $\begin{array}{l}\text { Harmful to pregnant } \\
\text { woman, toxic for people } \\
\text { with stomach diseases }\end{array}$ & 2 \\
\hline $\begin{array}{l}\text { Citrullus colocynthis } \\
\text { (L.) Schrad. }\end{array}$ & $\begin{array}{l}\text { H'adej } \\
\text { Handa'al }\end{array}$ & Herb/HUMPOM596 & $\begin{array}{c}\text { Internal and external } \\
\text { uses }\end{array}$ & $\begin{array}{c}\text { Cancer, diabetes, } \\
\text { detoxification, diuretic }\end{array}$ & Fruit & $\begin{array}{c}\text { Death (high doses), abortion, } \\
\text { diarrhea } \\
\text { vomiting }\end{array}$ & 9 \\
\hline \multicolumn{8}{|l|}{ Cupressaceae } \\
\hline $\begin{array}{l}\text { Tetraclinis articulata } \\
\text { (Vahl) Mast. }\end{array}$ & Ar-âr & Tree/HUMPOM597 & $\begin{array}{c}\text { Internal and external } \\
\text { uses }\end{array}$ & Colds, headache, skin diseases & Leaf & Neurotoxicity & 2 \\
\hline \multicolumn{8}{|l|}{ Ephedraceae } \\
\hline $\begin{array}{c}\text { Ephedra altissima } \\
\text { Desf. }\end{array}$ & Laâlenda & Shrub/HUMPOM598 & Internal uses & Cancer & Aerial part & Dizziness & 3 \\
\hline
\end{tabular}


Table 2. Cont.

\begin{tabular}{|c|c|c|c|c|c|c|c|}
\hline $\begin{array}{c}\text { Family } \\
\text { Scientific Name }\end{array}$ & Local Name & $\begin{array}{l}\text { Herbaceous Life } \\
\text { Form/Voucher Number }\end{array}$ & $\begin{array}{c}\text { Rout of } \\
\text { Administration }\end{array}$ & Traditional Uses & Poisonous Part & Toxic Signs & NC \\
\hline \multicolumn{8}{|l|}{ Euphorbiaceae } \\
\hline $\begin{array}{c}\text { Euphorbia resinifera } \\
\text { O. Berg } \\
\text { Fabaceae }\end{array}$ & $\begin{array}{l}\text { Takiwt } \\
\text { Takawt }\end{array}$ & Shrub/HUMPOM599 & $\begin{array}{c}\text { Internal and external } \\
\text { uses }\end{array}$ & Hair care, diabetes & Seed & Death & 2 \\
\hline $\begin{array}{l}\text { Glycine max (L.) } \\
\text { Merr. }\end{array}$ & Soja & $\begin{array}{c}\text { Herb/HUMPOM600 } \\
\text { Shrub/ }\end{array}$ & Internal uses & $\begin{array}{c}\text { To weight gain, against } \\
\text { menopause, appetite increases } \\
\text { female hormones, } \\
\text { hypertension colon }\end{array}$ & Seed & $\begin{array}{c}\text { Sterility, sedation, sleep, } \\
\text { diarrhea, blocks absorption } \\
\text { of nutrients (high dose), } \\
\text { colic, nausea, constipation, } \\
\text { hypertension }\end{array}$ & 2 \\
\hline $\begin{array}{l}\text { Retama monosperma } \\
\text { (L.) Boiss. }\end{array}$ & Rtem & HUMPOM601 & External uses & Magic, hypoglycemia & Stem & Death (high doses), abortion & 12 \\
\hline $\begin{array}{c}\text { Trigonella } \\
\text { foenum-graecum } \mathrm{L} .\end{array}$ & Lhelba & Herb/HUMPOM602 & Internal uses & $\begin{array}{l}\text { Anemia, appetite, weight gain, } \\
\text { blood detoxification, digestive } \\
\text { system, facilitate food } \\
\text { absorption, cold, antiparasitic }\end{array}$ & Seeds & $\begin{array}{c}\text { Jaundice, harmful to } \\
\text { pregnant woman, renal pain, } \\
\text { hypertension, } \\
\text { gastrointestinal diseases, } \\
\text { nausea, decreases immunity, } \\
\text { stomachache (in high doses), } \\
\text { abortion }\end{array}$ & 12 \\
\hline \multicolumn{8}{|l|}{ Gentianaceae } \\
\hline $\begin{array}{l}\text { Centaurium } \\
\text { erythraea Rafn. }\end{array}$ & $\begin{array}{l}\text { Gosset l-hayya, } \\
\text { Marraret al-hench }\end{array}$ & Herb/HUMPOM603 & Internal uses & Infertility, skin diseases & Seed & Nausea and dizziness & 2 \\
\hline \multicolumn{8}{|l|}{ Hypericaceae } \\
\hline $\begin{array}{c}\text { Hypericum perforatum } \\
\text { L. }\end{array}$ & Hachicht lkalb & Herb/HUMPOM604 & $\begin{array}{c}\text { Internal and external } \\
\text { uses }\end{array}$ & Asthma, varicose veins & Flower & $\begin{array}{l}\text { Death, nephrotoxicity, } \\
\text { dermatological problems }\end{array}$ & 4 \\
\hline
\end{tabular}


Table 2. Cont.

\begin{tabular}{|c|c|c|c|c|c|c|c|}
\hline $\begin{array}{c}\text { Family } \\
\text { Scientific Name }\end{array}$ & Local Name & $\begin{array}{c}\text { Herbaceous Life } \\
\text { Form/Voucher Number }\end{array}$ & $\begin{array}{c}\text { Rout of } \\
\text { Administration }\end{array}$ & Traditional Uses & Poisonous Part & Toxic Signs & NC \\
\hline \multicolumn{8}{|l|}{ Iridaceae } \\
\hline Crocus sativus L. & Za'fran lhor & Herb/HUMPOM605 & Internal uses & $\begin{array}{l}\text { Digestion, anemia, asthma, } \\
\text { cough }\end{array}$ & Stigma & $\begin{array}{l}\text { Hypotension, dry mouth, } \\
\text { allergic reaction (flushing of } \\
\text { the face) }\end{array}$ & 2 \\
\hline \multicolumn{8}{|l|}{ Lamiaceae } \\
\hline Mentha spicata L. & Naana'a & Herb/HUMPOM606 & $\begin{array}{c}\text { Internal and external } \\
\text { uses }\end{array}$ & $\begin{array}{l}\text { Stomachache, lose weight, } \\
\text { heart problems, cosmetic }\end{array}$ & $\begin{array}{c}\text { Leaf } \\
\text { Aerial part }\end{array}$ & Intestinal gas, colon bloating & 8 \\
\hline Salvia officinalis L. & Salmia & Shrub/HUMPOM607 & Internal & $\begin{array}{c}\text { Diabetes, balance female } \\
\text { hormones, painful menstrual } \\
\text { periods, influenza, pain killer, } \\
\text { digestive system }\end{array}$ & $\begin{array}{l}\text { Aerial part } \\
\text { Leaf }\end{array}$ & Fever, nausea & 5 \\
\hline \multicolumn{8}{|l|}{ Molluginaceae } \\
\hline Corrigiola litoralis $\mathrm{L}$. & Serghina & Herb/HUMPOM608 & External uses & Cosmetic & $\begin{array}{l}\text { Root } \\
\text { Leaf }\end{array}$ & $\begin{array}{l}\text { Diarrhea (oral), abdominal } \\
\text { pain }\end{array}$ & 2 \\
\hline Morus alba L. & Toute & Tree/HUMPOM609 & Internal uses & Anemia, cough & Fruit & $\begin{array}{c}\text { Mouth inflammation (high } \\
\text { doses) }\end{array}$ & 2 \\
\hline \multicolumn{8}{|l|}{ Myristicaceae } \\
\hline $\begin{array}{c}\text { Myristica fragrans } \\
\text { Houtt }\end{array}$ & Lgouza & Tree/HUMPOM610 & Internal uses & Food & Fruit & Dizziness & 2 \\
\hline \multicolumn{8}{|l|}{ Nitrariaceae } \\
\hline Peganum harmala L. & Harmel & Herb/HUMPOM611 & $\begin{array}{l}\text { Internal and external } \\
\text { uses }\end{array}$ & $\begin{array}{c}\text { Headache, cold, hair care, } \\
\text { constipation, fever, anorexia, } \\
\text { diarrhea, abortion, } \\
\text { lengthening hair, arthritis, } \\
\text { rheumatism, diabetes, magic, } \\
\text { bad spirit, facilitation of } \\
\text { childbirth }\end{array}$ & $\begin{array}{l}\text { Seed } \\
\text { Aerial part } \\
\text { Leaf }\end{array}$ & $\begin{array}{c}\text { Neurological disorders } \\
\text { vomiting, abortion } \\
\text { hepatoxicity, eye irritations, } \\
\text { death, dizziness }\end{array}$ & 23 \\
\hline
\end{tabular}


Table 2. Cont.

\begin{tabular}{|c|c|c|c|c|c|c|c|}
\hline $\begin{array}{c}\text { Family } \\
\text { Scientific Name }\end{array}$ & Local Name & $\begin{array}{c}\text { Herbaceous Life } \\
\text { Form/Voucher Number }\end{array}$ & $\begin{array}{c}\text { Rout of } \\
\text { Administration }\end{array}$ & Traditional Uses & Poisonous Part & Toxic Signs & NC \\
\hline \multicolumn{8}{|l|}{ Solanaceae } \\
\hline $\begin{array}{c}\text { Solanum sodomaeum } \\
\text { L. } \\
\text { Papaveraceae }\end{array}$ & Matichat lahmir & Shrub/HUMPOM612 & External uses & Rheumatism & Berries & Death & 2 \\
\hline Papaver somniferum $\mathrm{L}$. & Kherkhacha & Herb/HUMPOM613 & Internal uses & Calming, sedative & Fruit & Neurological disorders & 6 \\
\hline \multicolumn{8}{|l|}{ Ranunculaceae } \\
\hline Nigella sativa L. & Sanouj & Herb/HUMPOM614 & Internal uses & $\begin{array}{c}\text { Respiratory infections, cold, } \\
\text { diabetes, digestive disease, } \\
\text { muscle stiffness }\end{array}$ & Seed & $\begin{array}{l}\text { Dizziness (high doses), } \\
\text { irritations, hepatotoxicity }\end{array}$ & 5 \\
\hline \multicolumn{8}{|l|}{ Rhamnaceae } \\
\hline $\begin{array}{l}\text { Ziziphus lotus (L.) } \\
\text { Lam. }\end{array}$ & Sedr-Nbeg & Shrub/HUMPOM615 & Internal uses & Digestive disease, diabetes & Root & Bladder toxicity & 2 \\
\hline \multicolumn{8}{|l|}{ Rosaceae } \\
\hline \multicolumn{8}{|l|}{ Rutaceae } \\
\hline Citrus $x$ aurantium $\mathrm{L}$. & Ranje & Tree/HUMPOM617 & Internal uses & $\begin{array}{l}\text { Hypertension, fever, cod, } \\
\text { diarrhea, cosmetic, increase } \\
\text { blood flow and circulation }\end{array}$ & $\begin{array}{l}\text { Fruit } \\
\text { Leaf } \\
\text { pulp }\end{array}$ & $\begin{array}{l}\text { Hypoglycemia, abortion, } \\
\text { cardiac problem, } \\
\text { hypertension }\end{array}$ & 5 \\
\hline Ruta montana (L.) L. & Fijel & Herb/HUMPOM618 & $\begin{array}{l}\text { Internal and external } \\
\text { uses }\end{array}$ & $\begin{array}{l}\text { Diarrhea, headache, fever, } \\
\text { menstrual disorders, diabetes, } \\
\text { bad spirit }\end{array}$ & $\begin{array}{l}\text { Whole plant } \\
\text { Leaf } \\
\text { Aerial part }\end{array}$ & $\begin{array}{c}\text { Diarrhea, vomiting } \\
\text { Digestive disorders, nervous } \\
\text { disorder }\end{array}$ & 5 \\
\hline \multicolumn{8}{|l|}{ Santalaceae } \\
\hline Viscum album $\mathrm{L}$. & Lenjbar & Tree/HUMPOM619 & Internal uses & Diarrhea, throat disorders & Seed & $\begin{array}{l}\text { Constipation } \\
\text { Hepatotoxicity }\end{array}$ & 2 \\
\hline
\end{tabular}


Table 2. Cont.

\begin{tabular}{|c|c|c|c|c|c|c|c|}
\hline $\begin{array}{c}\text { Family } \\
\text { Scientific Name }\end{array}$ & Local Name & $\begin{array}{c}\text { Herbaceous Life } \\
\text { Form/Voucher Number }\end{array}$ & $\begin{array}{c}\text { Rout of } \\
\text { Administration }\end{array}$ & Traditional Uses & Poisonous Part & Toxic Signs & NC \\
\hline \multicolumn{8}{|l|}{ Solanaceae } \\
\hline $\begin{array}{c}\text { Atropa } \\
\text { Belladonna L. }\end{array}$ & Zbib lidur & Herb/HUMPOM620 & Internal uses & Stimulant and aphrodisiac, & $\begin{array}{l}\text { Berries } \\
\text { seed }\end{array}$ & $\begin{array}{l}\text { Nervous disorder, colic, } \\
\text { gastroenteritis, death }\end{array}$ & 5 \\
\hline $\begin{array}{l}\text { Datura stramonium } \\
\text { L. }\end{array}$ & Chdeq jmel & Herb/HUMPOM621 & Internal uses & $\begin{array}{l}\text { Headache, menstrual blood, } \\
\text { sexual stimulant, sedative }\end{array}$ & Seed & $\begin{array}{l}\text { Delirium, madness, } \\
\text { hallucination }\end{array}$ & 4 \\
\hline $\begin{array}{c}\text { Hyoscyamus albus L. } \\
\text { Thymelaeaceae }\end{array}$ & Bounarjouf & Herb/HUMPOM622 & $\begin{array}{c}\text { Internal and external } \\
\text { uses }\end{array}$ & $\begin{array}{c}\text { Skin infections, eczema, } \\
\text { sedative }\end{array}$ & $\begin{array}{c}\text { Seed } \\
\text { Aerial part }\end{array}$ & Dizziness, hallucination & 2 \\
\hline $\begin{array}{l}\text { Daphne gnidium } \\
\text { L. }\end{array}$ & Lezzâz & Herb/HUMPOM623 & external uses & $\begin{array}{c}\text { Hair hydration, hair } \\
\text { elongation }\end{array}$ & $\begin{array}{c}\text { Leaf } \\
\text { Aerial part }\end{array}$ & $\begin{array}{c}\text { Dizziness, fainting } \\
\text { eye irritation, skin problems }\end{array}$ & 10 \\
\hline \multicolumn{8}{|l|}{ Euphorbiaceae } \\
\hline Ricinus communis L. & Kherouaa & Tree/HUMPOM624 & $\begin{array}{c}\text { Internal and external } \\
\text { uses }\end{array}$ & $\begin{array}{l}\text { Abdominal pain, constipation, } \\
\text { cosmetic (hair and face), } \\
\text { detoxification, eyelash } \\
\text { strengthening }\end{array}$ & Seed (oil) & $\begin{array}{l}\text { Vomiting, eye pain, death, } \\
\text { nausea }\end{array}$ & 10 \\
\hline Urtica dioica $\mathrm{L}$. & Lhoriga & Herb/HUMPOM625 & $\begin{array}{c}\text { Internal and external } \\
\text { uses }\end{array}$ & $\begin{array}{l}\text { Menstrual pain, respiratory } \\
\text { system } \\
\text { diabetes, rheumatism, cold, } \\
\text { kidneys }\end{array}$ & $\begin{array}{l}\text { Aerial part } \\
\quad \text { Leaf }\end{array}$ & $\begin{array}{c}\text { skin irritation, allergy, } \\
\text { stomachache, skin problems }\end{array}$ & 8 \\
\hline
\end{tabular}


Table 3. Category of illness and their informant consensus factor (ICF).

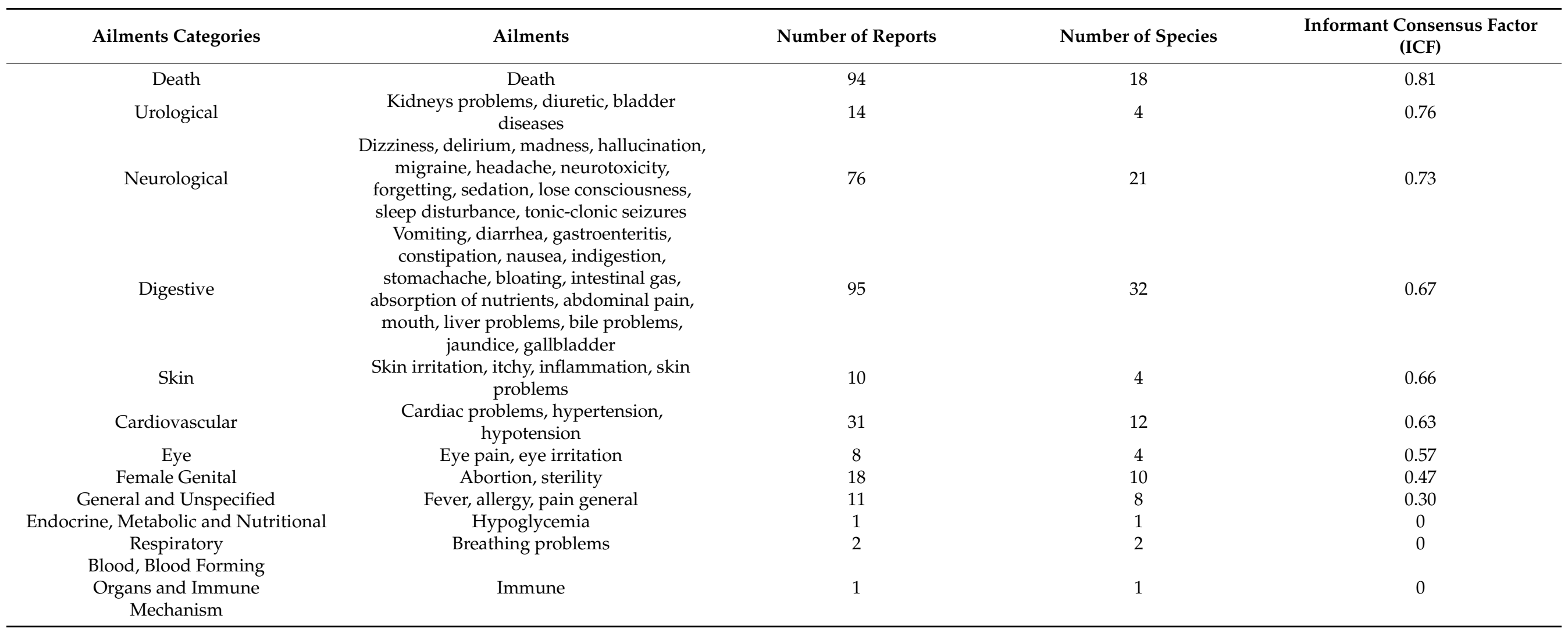




\subsubsection{Fidelity Level (FL)}

In Table 4, we regrouped the toxic plant species according to their corresponding fidelity level. Our results showed that the value of the fidelity level of plant species for a specific disease varied between 3.84 and $100 \%$.

- Concerning the death category, the most important species were Solanum sodomaeum L. $(\mathrm{Fl}=100 \%)$, Nerium oleander $\mathrm{L} .(\mathrm{Fl}=77.14 \%)$, and Citrullus colocynthis (L.) Schrad. $(\mathrm{Fl}=66 \%)$.

- $\quad$ For the digestive category, Mentha spicata L. $(\mathrm{Fl}=100 \%)$, Arisarum vulgare O. Targ. Tozz. $(\mathrm{Fl}=100 \%)$, Morus alba $\mathrm{L} .(\mathrm{Fl}=100 \%)$, Viscum album $\mathrm{L} .(\mathrm{Fl}=100 \%)$, and Ruta montana (L.) L. ( $\mathrm{Fl}=80 \%)$.

- The most common plants in the cardiovascular category were Petroselinum crispum (Mill.) Fuss $(\mathrm{Fl}=100 \%)$, Citrus $x$ aurantium $\mathrm{L} .(\mathrm{Fl}=80 \%)$, and Salvia officinalis L. $(\mathrm{Fl}=80 \%)$.

- For the neurological category, the plant with the highest Fl $(100 \%)$ were Ephedra altissima Desf., Papaver somniferum L., Datura stramonium L., and Anastatica hierochuntica L.

- Crocus sativus L. was reported with $\mathrm{Fl}$ of $100 \%$ for the general and unspecified category.

- $\quad$ For the skin category, we found Urtica dioica $\mathrm{L}$. $(\mathrm{Fl}=87.5 \%)$ was the most important.

- $\quad$ For the female genital category, Glycine max (L.) Merr. $(\mathrm{Fl}=50 \%$ ) was the most important.

The toxic species with high fidelity level constitute the most important toxic herbs cited by the informants, causing a particular illness category. Furthermore, the plants causing repetitive are more likely to have some toxic compounds. For the plants with low fidelity level, we can note the following species:

- Peganum harmala L. and Cannabis sativa L. for the digestive category;

- Nerium oleander L. for the cardiovascular and eye categories;

- Glycine max (L.) Merr. for the neurological category;

- Peganum harmala L. for the female genital category;

- $\quad$ Allium satioum L. for the skin category;

- Carlina gummifera (L.) Less. and Cannabis sativa L. for the respiratory category; and

- Trigonella foenum-graecum L. for endocrine, metabolic and nutritional, blood, blood forming organs, and immune mechanism.

These plants may cause little harm. Furthermore, the low ICF value observed in our study could be attributed to a lack of communication among people working in various areas of study as previously hypothesized [17].

Table 4. The plants with fidelity level values.

\begin{tabular}{ccc}
\hline Category of Illness & Name of Species & Fidelity Level (Fl \%) \\
\hline & Solanum sodomaeum L. & $100.00 \%$ \\
& Nerium oleander L. & $77.14 \%$ \\
& Citrullus colocynthis (L.) Schrad. & $66.00 \%$ \\
& Carlina gummifera (L.) Less. & $64.00 \%$ \\
Death & Prunus dulcis (Mill.) D.A.Webb & $60.00 \%$ \\
& Ferula communis L. & $57.14 \%$ \\
& Aristolochia fontanesii Boiss. \& Reut. & $40.00 \%$ \\
& Cannabis sativa L. & $34.61 \%$ \\
& Drimia maritima (L.) Stearn & $33.33 \%$ \\
& Hypericum perforatum L. & $20.00 \%$ \\
& Peganum harmala L. & $17.39 \%$ \\
\hline
\end{tabular}


Table 4. Cont.

\begin{tabular}{|c|c|c|}
\hline Category of Illness & Name of Species & Fidelity Level (Fl \%) \\
\hline Digestive & $\begin{array}{c}\text { Mentha spicata L. } \\
\text { Arisarum vulgare O. Targ. Tozz. } \\
\text { Morus alba L. } \\
\text { Viscum album L. } \\
\text { Ruta montana (L.) L. } \\
\text { Thapsia garganica L. } \\
\text { Haloxylon scoparium Pomel } \\
\text { Artemisia arborescens (Vaill.) L. } \\
\text { Bryonia cretica subsp. dioica (Jacq.) Tutin } \\
\text { Pistacia lentiscus L. } \\
\text { Ricinus communis L. } \\
\text { Drimia maritima (L.) Stearn } \\
\text { Carlina gummifera (L.) Less. } \\
\text { Glycine max (L.) Merr. } \\
\text { Citrullus colocynthis (L.) Schrad. } \\
\text { Allium sativum L. } \\
\text { Conium maculatum L. } \\
\text { Trigonella foenum-graecum L. } \\
\text { Aristolochia fontanesii Boiss. \& Reut. } \\
\text { Salvia officinalis L. } \\
\text { Nigella sativa } \text { L. } \\
\text { Nerium oleander L. } \\
\text { Lepidium sativum L. } \\
\text { Urtica dioica L. } \\
\text { Peganum harmala L. } \\
\text { Cannabis sativa L. }\end{array}$ & $\begin{array}{c}100.00 \% \\
100.00 \% \\
100.00 \% \\
100.00 \% \\
80.00 \% \\
75.00 \% \\
66.66 \% \\
50.00 \% \\
50.00 \% \\
50.00 \% \\
50.00 \% \\
50.00 \% \\
44.00 \% \\
41.66 \% \\
33.00 \% \\
31.18 \% \\
25.00 \% \\
25.00 \% \\
20.00 \% \\
20.00 \% \\
20.00 \% \\
17.14 \% \\
12.50 \% \\
12.50 \% \\
8.69 \% \\
7.69 \%\end{array}$ \\
\hline Cardiovascular & $\begin{array}{l}\text { Petroselinum crispum (Mill.) Fuss } \\
\text { Citrus x aurantium L. } \\
\text { Salvia officinalis L. } \\
\text { Allium satioum L. } \\
\text { Nigella sativa L. } \\
\text { Prunus dulcis (Mill.) D.A.Webb } \\
\text { Trigonella foenum-graecum L. } \\
\text { Lepidium sativum L. } \\
\text { Nerium oleander L. }\end{array}$ & $\begin{array}{c}100.00 \% \\
80.00 \% \\
80.00 \% \\
50.00 \% \\
20.00 \% \\
20.00 \% \\
16.66 \% \\
12.50 \% \\
2.85 \%\end{array}$ \\
\hline Neurological & $\begin{array}{c}\text { Ephedra altissima Desf. } \\
\text { Papaver somniferum L. } \\
\text { Datura stramonium L. } \\
\text { Anastatica hierochuntica L. } \\
\text { Coriandrum sativum L. } \\
\text { Conium maculatum L. } \\
\text { Peganum harmala L. } \\
\text { Cannabis sativa L. } \\
\text { Daphne gnidium L. } \\
\text { Nigella sativa L. } \\
\text { Artemisia arborescens (Vaill.) L. } \\
\text { Lepidium sativum L. } \\
\text { Ruta montana (L.) L. } \\
\text { Drimia maritima (L.) Stearn } \\
\text { Ferula communis L. } \\
\text { Glycine max (L.) Merr. }\end{array}$ & $\begin{array}{c}100.00 \% \\
100.00 \% \\
100.00 \% \\
100.00 \% \\
80.00 \% \\
75.00 \% \\
69.56 \% \\
61.53 \% \\
60.00 \% \\
60.00 \% \\
50.00 \% \\
37.00 \% \\
20.00 \% \\
16.66 \% \\
28.57 \% \\
8.33 \%\end{array}$ \\
\hline
\end{tabular}


Table 4. Cont.

\begin{tabular}{|c|c|c|}
\hline Category of Illness & Name of Species & Fidelity Level (F1 \%) \\
\hline \multirow{9}{*}{ Female Genitals } & Glycine max (L.) Merr. & $50.00 \%$ \\
\hline & Citrus $x$ aurantium $\mathrm{L}$. & $40.00 \%$ \\
\hline & Thapsia garganica L. & $25.00 \%$ \\
\hline & Trigonella foenum-graecum L. & $25.00 \%$ \\
\hline & Bryonia cretica subsp. dioica (Jacq.) Tutin & $20.00 \%$ \\
\hline & Ferula communis L. & $14.28 \%$ \\
\hline & Lepidium satioum $\mathrm{L}$. & $12.50 \%$ \\
\hline & Citrullus colocynthis (L.) Schrad. & $11.00 \%$ \\
\hline & Peganum harmala L. & $8.69 \%$ \\
\hline \multirow{5}{*}{ Urological } & Aristolochia fontanesii Boiss. \& Reut. & $46.00 \%$ \\
\hline & Hypericum perforatum L. & $25.00 \%$ \\
\hline & Trigonella foenum-graecum L. & $16.66 \%$ \\
\hline & Lepidium sativum $\mathrm{L}$. & $12.50 \%$ \\
\hline & Allium sativum $\mathrm{L}$. & $4.54 \%$ \\
\hline \multirow{3}{*}{ Skin } & Urtica dioica $\mathrm{L}$. & $87.50 \%$ \\
\hline & Pistacia lentiscus L. & $50.00 \%$ \\
\hline & Daphne gnidium L. & $10.00 \%$ \\
\hline \multirow{7}{*}{ General and Unspecified } & Crocus satious L. & $100.00 \%$ \\
\hline & Haloxylon scoparium Pomel & $33.33 \%$ \\
\hline & Coriandrum satioum L. & $20.00 \%$ \\
\hline & Salvia officinalis L. & $20.00 \%$ \\
\hline & Prunus dulcis (Mill.) D.A.Webb & $20.00 \%$ \\
\hline & Ferula communis L. & $14.28 \%$ \\
\hline & Allium sativum $\mathrm{L}$. & $13.63 \%$ \\
\hline \multirow{3}{*}{ Eye } & Daphne gnidium L. & $30.00 \%$ \\
\hline & Ricinus communis $\mathrm{L}$. & $20.00 \%$ \\
\hline & Nerium oleander L. & $2.85 \%$ \\
\hline \multirow{2}{*}{ Respiratory } & Carlina gummifera (L.) Less. & $4.00 \%$ \\
\hline & Cannabis sativa $\mathrm{L}$. & $3.84 \%$ \\
\hline \multirow{2}{*}{ Endocrine, Metabolic, and Nutritional } & Citrus $x$ aurantium $L$ & $20.00 \%$ \\
\hline & Trigonella foenum-graecum L. & $8.33 \%$ \\
\hline Blood, Blood Forming & & \\
\hline $\begin{array}{c}\text { Organs, and Immune } \\
\text { Mechanism }\end{array}$ & Trigonella foenum-graecum L. & $8.33 \%$ \\
\hline
\end{tabular}

\section{Materials and Methods}

\subsection{Study Area}

The study zone is located in the northeastern region of Morocco (Figure 3). This part of the country is one of the twelve regions of Morocco including eight province districts with a total of $90,130 \mathrm{~km}^{2}$ (12\% of the national territory). According to the national census report published in 2014, the population in this region reached 2,314,346 (6.8\% of the national population) with a density of 26 persons per square kilometer [33]. The dominant language in this region was the dialect Arabic. In the second position, we found the Berber language, which is subdivided into two dialects, with low proportions: Tarifit in the north of the region and Tashelhit in the south. The climatic variability in the region extends from the north to the Saharan in the south. Because of these characteristics, the region has a high level of biodiversity including a diverse flora and, in particular, medicinal plants. 

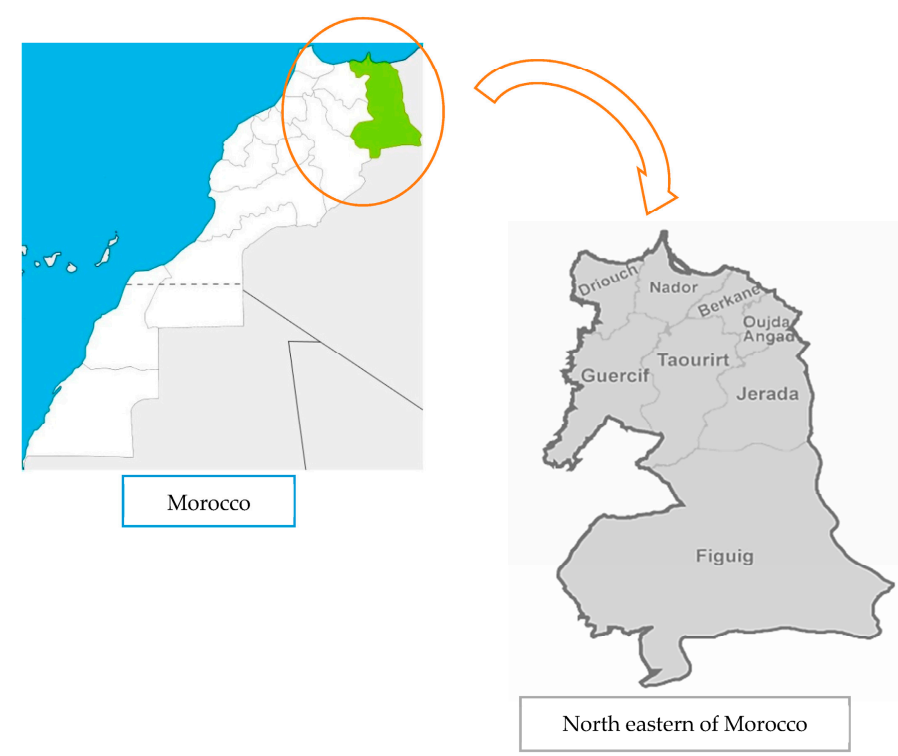

Figure 3. Geographical position of northeastern Morocco.

\subsection{Ethnobotanical Data Collection}

From April 2017 to June 2019, a fieldwork survey was carried out in order to lay down a thorough ethnobotanical study of poisonous and potential poisonous species with significant toxicity effects. Ethnobotanical data were collected from 386 informants aged 20 and up from various districts in northeastern Morocco (Table 1). The study area included the Oujda-Angad, Berkane, and Jerada districts. These areas were selected because of their cultural and botanical diversities. The sampling technique used in this ethnobotanical investigation was simple random sampling. The data were obtained through structured and semi-structured interviews and face-to-face conversations with local inhabitants and herbalists of the study areas. Permission were obtained from the interviewees prior to the interview. The questionnaires were divided into two sections: the first focused on informant profiles such as location, age, gender, and academic level, while the second collected ethnobotanical data about the plants including local names, traditional uses, administration route, toxic part, and toxic signs. Only plants that were mentioned more than once were selected for analysis.

\subsection{Plant Collection and Identification}

The names of the plant species mentioned by the interviewees were identified using technical documentation related to Moroccan flora [34-36] and compared with the specimens already in the Herbarium of Mohammed First University Oujda-Morocco (HUMPOM). A voucher number was assigned to each specimen and deposited in the HUMPOM. The scientific names of plants selected were checked against The Plant List Database [37].

\subsection{Analysis of Ethnomedicinal Data}

The data recorded on the questionnaire sheets during the survey trips were sorted in an Excel 2016 sheet and analyzed for quantitative indices like the informant consensus factor (ICF) and fidelity level (Fl).

\subsubsection{Informant Consensus Factor (ICF)}

The informant consensus factor (ICF) was initially used to determine whether respondents agreed on the use of plants to treat various ailments. This parameter was used in this study, with minor modifications, to determine the informants' agreement on the toxicity of selected species.

The ICF was calculated using the following formula [38]: 
$\mathrm{ICF}=\mathrm{Nur}-\mathrm{Nt} / \mathrm{Nur}-1$, where Nur refers to the number of reports for a particular ailment category, and $\mathrm{Nt}$ refers to the number of plants affected by this particular ailment category. This index can only have a value between 0 and 1 . A high value (close to 1 ) indicated that the toxicity of the revealed plants is primarily known by the informants and/or how the toxicity of the plants is exchanged between respondents. A low value of this parameter, on the other hand, indicated that the informants disagreed on the toxicity of specific plants or that the respondents did not exchange their knowledge about the toxicity [39-41].

\subsubsection{Fidelity level (FL)}

The fidelity level (Fl) of each plant was calculated as commonly adopted [42]:

Fl $(\%)=(\mathrm{Ns} / \mathrm{N}) \times 100$, where $\mathrm{Ns}$ is the number of respondents who informed the toxicity of a specific plant for specific toxicity, and $\mathrm{N}$ is the total number of informants who informed all significant toxicities of the plant. The plants mentioned once were excluded from this analysis.

\section{Conclusions}

In total, fifty-five medicinal plants belonging to 36 families were here reported to be toxic or present a potential toxicity by indigenous people from northeastern Morocco. These results indicated that this population has extensive traditional knowledge of medicinal plants and their harmful effects, and quantitative analyses showed that the inventoried plants may negatively impact different organ and pose a risk to human health. Despite the fact that some of the reported plants as used commonly in culinary and acute health problems, the dose is a key factor defining where the effect tends to be therapeutic or toxic. Caution should be exercised when using these plants, particularly for medicinal purposes, and adequate information on these plants including toxicity, composition, and safe doses should be obtained. In the present study, the FI and ICF results could be followed to presume the toxicity (high FI and ICF) or the potential toxicity (low FI and ICF). In accordance with this, it is suggested that additional studies be conducted (i) to confirm traditional information associated with poisonous plants using appropriate experiments, and (ii) to determine the identity of toxic phytochemicals associated with poisonous plants.

Author Contributions: Conceptualization, L.K. and M.E.; Methodology, L.K. and N.B.; Validation, M.E.; Formal analysis and critical reading, M.A. and C.H.; Revision, L.K. and M.B.; Data curation, H.M.; Writing—original draft preparation, L.K..; Supervision, M.E; All authors have read and agreed to the published version of the manuscript.

Funding: This research received no external funding.

Institutional Review Board Statement: Not applicable.

Informed Consent Statement: Informed consent was obtained from all subjects involved in the study.

Data Availability Statement: Data are available from the authors upon reasonable request.

Conflicts of Interest: The authors declare no conflict of interest.

\section{References}

1. Perrino, E.V.; Valerio, F.; Gannouchi, A.; Trani, A. Ecological and Plant Community Implication on Essential Oils Composition in Useful Wild Officinal Species: A Pilot Case Study in Apulia (Italy). Plants 2021, 10, 574. [CrossRef]

2. Singh, B.; Singh, B.; Kishor, A.; Singh, S.; Bhat, M.N.; Surmal, O.; Musarella, C.M. Exploring Plant-Based Ethnomedicine and Quantitative Ethnopharmacology: Medicinal Plants Utilized by the Population of Jasrota Hill in Western Himalaya. Sustainability 2020, 12, 7526. [CrossRef]

3. Aziz, M.A.; Ullah, Z.; Pieroni, A. Wild Food Plant Gathering among Kalasha, Yidgha, Nuristani and Khowar Speakers in Chitral, NW Pakistan. Sustainability 2020, 12, 9176. [CrossRef]

4. Omwenga, E.O.; Hensel, A.; Shitandi, A.; Goycoolea, F.M. Ethnobotanical Survey of Traditionally Used Medicinal Plants for Infections of Skin, Gastrointestinal Tract, Urinary Tract and the Oral Cavity in Borabu Sub-County, Nyamira County, Kenya. J. Ethnopharmacol. 2015, 176, 508-514. [CrossRef] [PubMed] 
5. El-hilaly, J.; Hmammouchi, M.; Lyoussi, B. Ethnobotanical Studies and Economic Evaluation of Medicinal Plants in Taounate Province (Northern Morocco). J. Ethnopharmacol. 2003, 86, 149-158. [CrossRef]

6. Tahraoui, A.; El-hilaly, J.; Israili, Z.H.; Lyoussi, B. Ethnopharmacological Survey of Plants Used in the Traditional Treatment of Hypertension and Diabetes in South-Eastern Morocco (Errachidia Province). J. Ethnopharmacol. 2007, 110, 105-117. [CrossRef]

7. Eddouks, M.; Maghrani, M.; Lemhadri, A.; Ouahidi, M.; Jouad, H. Ethnopharmacological Survey of Medicinal Plants Used for the Treatment of Diabetes Mellitus, Hypertension and Cardiac Diseases in the South-East Region of Morocco (Tafilalet). J. Ethnopharmacol. 2002, 82, 97-103. [CrossRef]

8. Bellakhdar, J.; Claisse, R.; Fleurentin, J.; Younos, C. Repertory of Standard Herbal Drugs in the Moroccan Pharmacopoea. J. Ethnopharmacol. 1991, 35, 123-143. [CrossRef]

9. Alami Merrouni, I.; Elachouri, M. Anticancer Medicinal Plants Used by Moroccan People: Ethnobotanical, Preclinical, Phytochemical and Clinical Evidence. J. Ethnopharmacol. 2021, 266, 113435. [CrossRef]

10. Fakchich, J.; Elachouri, M. Ethnobotanical Survey of Medicinal Plants Used by People in Oriental Morocco to Manage Various Ailments. J. Ethnopharmacol. 2014, 154, 76-87.

11. Ziyyat, A.; Legssyer, A.; Mekhfi, H.; Dassouli, A.; Serhrouchni, M. Phytotherapy of Hypertension and Diabetes in Oriental Morocco. J. Ethnopharmacol. 1997, 58, 45-54. [CrossRef]

12. Mordeniz, C. Integration of Traditional and Complementary Medicine into Evidence-Based Clinical Practice. In Traditional and Complementary Medicine; IntechOpen: London, UK, 2019; pp. 1-8.

13. Kharchoufa, L.; Bouhrim, M.; Bencheikh, N.; El Assri, S.; Amirou, A.; Yamani, A.; Choukri, M.; Mekhfi, H.; Elachouri, M. Acute and subacute toxicity studies of the aqueous extract from Haloxylon scoparium pomel (Hammada scoparia (pomel)) by oral administration in Rodents. BioMed Res. Int. 2020. [CrossRef] [PubMed]

14. Ekor, M. The Growing Use of Herbal Medicines: Issues Relating to Adverse Reactions and Challenges in Monitoring Safety. Front. Neurol. 2014, 4, 177. [CrossRef] [PubMed]

15. Kharchoufa, L.; Merrouni, I.A.; Yamani, A.; Elachouri, M. Profile on Medicinal Plants Used by the People of North Eastern Morocco: Toxicity Concerns. Toxicon 2018, 154, 90-113. [CrossRef] [PubMed]

16. Najem, M.; Daoudi, A.; Bouiamrine, E.H.; Ibijbijen, J.; Nassiri, L. Biodiversity of Poisonous Medicinal Plants Solicited in the Traditional Phytotherapy of the Central Middle Atlas -Morocco. Ethnobot. Res. Appl. 2019, 18, 1-22. [CrossRef]

17. Bhatia, H.; Manhas, R.K.; Kumar, K.; Magotra, R. Traditional Knowledge on Poisonous Plants of Udhampur District of Jammu and Kashmir, India. J. Ethnopharmacol. 2014, 152, 207-216. [CrossRef]

18. Yahyaoui, O.; El Ouaaziz, N.A.; Sammama, A.; Kerrouri, S.; Bouabid, B.; Lrhorfi, L.A.; Zidane, L.; Bengueddour, R. Etude Ethnobotanique: Plantes Médicinales Commercialisées à La Province de Laâyoune; Identification et Utilisation. Int. J. Innov. Appl. Stud. 2015, 12, 533-541.

19. Musa, M.S.; Abdelrasool, F.E.; Elsheikh, E.A.; Ahmed, L.A.M.N.; Mahmoud, A.L.E.; Yagi, S.M. Ethnobotanical Study of Medicinal Plants in the Blue Nile State, South-Eastern Sudan. J. Med. Plants Res. 2011, 5, 4287-4297.

20. Eddouks, M.; Ajebli, M.; Hebi, M. Ethnopharmacological Survey of Medicinal Plants Used in Daraa-Tafilalet Region (Province of Errachidia), Morocco. J. Ethnopharmacol. 2017, 198, 516-530. [CrossRef] [PubMed]

21. Barkaoui, M.; Katiri, A.; Boubaker, H.; Msanda, F. Ethnobotanical Survey of Medicinal Plants Used in the Traditional Treatment of Diabetes in Chtouka Ait Baha and Tiznit (Western Anti-Atlas), Morocco. J. Ethnopharmacol. 2017, 198, 338-350. [CrossRef]

22. Mechchate, H.; Es-safi, I.; Jawhari, F.z.; Bari, A.; Grafov, A.; Bousta, D. Ethnobotanical Survey about the Management of Diabetes with Medicinal Plants Used by Diabetic Patient in Region of Fez-Meknes, Morocco. Ethnobot. Res. Appl. 2020, 19. [CrossRef]

23. Rodríguez, J.F.; López, A.V.; Núñez, D.R.; Franzi, A.V.; Castro, C.O. De Investigación y Divulgación Del Conocimiento Etnobiológico En Castilla-La Mancha. Sabuco Rev. Estud. Albacet. 2008, 6, 137-156.

24. Bellakhdar, J. La Pharmacopee Marocaine Traditionnelle: Medecine Arabe Ancienne et Savoirs Populaires; IBIS Press: Newburyport, MA, USA, 1997.

25. Thomas, E.; Vandebroek, I.; Sanca, S.; Van Damme, P. Cultural Significance of Medicinal Plant Families and Species among Quechua Farmers in Apillapampa, Bolivia. J. Ethnopharmacol. 2009, 122, 60-67. [CrossRef]

26. Huai, H.; Dong, Q.; Liu, A. Ethnomedicinal Analysis of Toxic Plants from Five Ethnic Groups in China. Ethnobot. Res. Appl. 2010, 8, 169-179. [CrossRef]

27. Pomilio, A.B.; Falzoni, E.M.; Vitale, A.A. Toxic Chemical Compounds of the Solanaceae. Nat. Prod. Commun. 2008, 3 , 593-628. [CrossRef]

28. Al-qura, S. Ethnobotanical Survey of Folk Toxic Plants in Southern Part of Jordan. Toxicon 2005, 46, 119-129. [CrossRef]

29. Bnouham, M.; Zahra Merhfour, F.; Elachoui, M.; Legssyer, A.; Mekhfi, H.; Lamnaouer, D.; Ziyyat, A. Toxic Effects of Some Medicinal Plants Used in Moroccan Traditional Medicine. Moroc. J. Biol. 2006, 23, 21-30.

30. Yamani, A.; Bunel, V.; Antoine, M.; Husson, C.; Stévigny, C.; Duez, P.; Elachouri, M.; Nortier, J. Substitution between Aristolochia and Bryonia Genus in North-Eastern Morocco: Toxicological Implications. J. Ethnopharmacol. 2015, 166, 250-260. [CrossRef]

31. Audi, J.; Belson, M.; Patel, M.; Schier, J.; Osterloh, J. Ricin Poisoning. JAMA 2005, 294, 2342-2351. [CrossRef]

32. WHO. International Classification of Primary Care, Second Edition (ICPC-2). Available online: https://www.who.int/ classifications/icd/adaptations/icpc2/en/ (accessed on 15 January 2020).

33. RGPH. Recensement Général de La Population et de l'Habitat Au. 2014. Available online: https://www.rgph2014.hcp.ma (accessed on 25 November 2020). 
34. Fennane, M.; Ibn Tatou, M.; Mathez, J.; Ouyahya, A.; El Oualidi, J. Flore Pratique Du Maroc. Manuel de Determination Des Plantes Vasculaires. Vol 1. Ptéridophyta, Gymnospermae, Angiospermae(Lauraceae, Neuradaceae); Travaux de l'institut Scientifique Série Botanique: Rabat, Morocco, 1999.

35. Fennane, M.; Ibn Tattou, M.; Ouyahya, A.; El Oualidi, J. Flore Pratique Du Maroc. Manuel de Determination Des Plantes Vasculaires. Vol. 2-Angiospermae (Leguminosae-Lentibulariaceae); Travaux de l'institut Scientifique Série Botanique: Rabat, Morocco, 2007.

36. Fennane, M.; Tattou, M.I.; Ouyahya, A.; El Oualidi, J. Flore Pratique Du Maroc. Manuel de Determination Des Plantes Vasculaires. Vol. 3-Dicotylédones (p.p),Monocotylédones; Travaux de l'institut Scientifique Série Botanique: Rabat, Morocco, 2014.

37. The Plant Lis. Available online: thttp:/ / www.theplantlist.org/ (accessed on 11 December 2020).

38. Heinrich, M.; Ankli, A.; Frei, B.; Weimann, C.; Sticher, O. Medicinal Plants in Mexico: Healers' Consensus and Cultural Importance. Soc. Sci. Med. 1998, 47, 1859-1871. [CrossRef]

39. Gazzaneo, L.R.S.; de Lucena, R.F.P.; de Albuquerque, U.P. Knowledge and Use of Medicinal Plants by Local Specialists in an Region of Atlantic Forest in the State of Pernambuco (Northeastern Brazil). J. Ethnobiol. Ethnomed. 2005, 1, 1-8. [CrossRef]

40. Srithi, K.; Balslev, H.; Wangpakapattanawong, P.; Srisanga, P.; Trisonthi, C. Medicinal Plant Knowledge and Its Erosion among the Mien (Yao) in Northern Thailand. J. Ethnopharmacol. 2009, 123, 335-342. [CrossRef] [PubMed]

41. Sharma, R.; Manhas, R.K.; Magotra, R. Ethnoveterinary Remedies of Diseases among Milk Yielding Animals in Kathua, Jammu and Kashmir, India. J. Ethnopharmacol. 2012, 141, 265-272. [CrossRef] [PubMed]

42. Friedman, J.; Yaniv, Z.; Dafnib, A.; Palewitcha, D. A Preliminary Classification of the Healing Potential of Medicinal Plants, Based on a Rational Analysis of an Ethnopharmacological Field Survey among Bedouins in the Negev Desert, Israel. J. Ethnopharmacol. 1986, 16, 275-287. [CrossRef] 